\title{
Detection of tyrosinase mRNA in the sentinel lymph nodes of melanoma patients is not a predictor of short-term disease recurrence
}

\author{
Cuneyt Tatlidil ${ }^{1}$, Winston S Parkhill², Carman A Giacomantonio ${ }^{2}$, Wenda L Greer ${ }^{1}$, \\ Steven F Morris ${ }^{2}$ and Noreen MG Walsh ${ }^{1}$ \\ ${ }^{1}$ Department of Pathology, Capital District Health Authority, Halifax, NS, Canada; ${ }^{2}$ Department of Surgery, \\ Capital District Health Authority, Halifax, NS, Canada
}

\begin{abstract}
Sentinel lymph node evaluation has enabled identification of patients with cutaneous melanoma who might benefit from elective regional lymph node dissection. Sentinel nodes are currently assessed by histologic and reverse transcription polymerase chain reaction (RT-PCR) evaluation for melanocyte-specific markers. The clinical significance of positive findings by RT-PCR in the absence of histologic evidence of metastasis (HIS ${ }^{\text {NEG/ }}$ $\mathrm{PCR}^{\mathrm{POS}}$ ) remains unclear. Examination of 264 lymph nodes from 139 patients revealed histopathologic positivity in 34 patients $(24.5 \%)$, in which 26 also demonstrated simultaneous RT-PCR positivity (HIS ${ }^{\text {POS }} /$ PCR $^{\text {POS }}$ ). Of 35 HIS $^{\text {NEG/PCR }}{ }^{\text {POS }}$ patients (25.2\%), five also had nodal capsular nevi. In total, capsular nevi were detected in 13 patients $(9.4 \%)$. A total of 70 patients $(50.4 \%)$ had negative sentinel nodes by both histopathology and RT-PCR (HIS $^{\text {NEG }} /$ PCR $^{\text {NEG }}$ ). Over a median follow-up of 25 months, local and/or systemic recurrence developed in 31 patients $(22.3 \%)$. Recurrence rates were similar among patients with histopathologic evidence of sentinel lymph node metastasis, irrespective of RT-PCR status (HIS ${ }^{\text {POS }} /$ PCR $^{\text {POS }} 62 \%$; HIS ${ }^{\text {POS }} /$ PCR $^{\text {NEG }} 75 \%$ ). In contrast, only $10 \%$ of HIS $^{\text {NEG }} / P_{C R}{ }^{\text {NEG }}$ patients developed recurrence, significantly less than those in either HIS ${ }^{\text {POS }}$ group

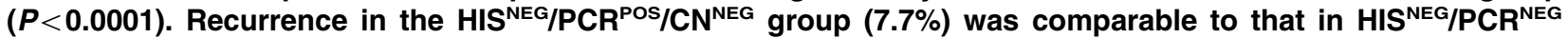
patients and significantly lower than that in either HIS ${ }^{\text {POS }}$ group $(P<0.0001)$. The only independent prognostic factors identified by multivariate analysis were the Breslow thickness of the primary tumour and histopathologic positivity of sentinel nodes. Our findings support previous observations that histopathologic evidence of metastatic melanoma in sentinel lymph nodes is an independent predictor of disease recurrence. In contrast, detection of tyrosinase mRNA by RT-PCR alone does not appear to increase the likelihood of shortterm disease recurrence.
\end{abstract}

Modern Pathology (2007) 20, 427-434. doi:10.1038/modpathol.3800754; published online 2 March 2007

\section{Introduction}

Lymph node status is one of the most important prognostic factors in cutaneous malignant melanoma. According to the 2002 American Joint committee on Cancer (AJCC) staging for cutaneous melanoma, the presence of regional nodal metastases (Stage III patients) has a significant adverse impact on survival when compared to node negative (Stages I and II) patients, ${ }^{1}$ emphasizing the need for early and precise assessment of regional lymph nodes.

Correspondence: Dr NMG Walsh, MD, QEII Health Sciences Centre, Division of Anatomical Pathology, 5788 University Avenue, Halifax, NS, Canada B3H 2 Y9.

E-mail: noreen.walsh@cdha.nshealth.ca

Received 23 October 2006; revised 03 January 2007; accepted 04 January 2007; published online 2 March 2007
The introduction of sentinel lymph node excision and evaluation over a decade ago has improved the accuracy of disease staging and this procedure is used routinely in the investigation of patients with intermediate thickness melanomas and no clinical evidence of metastasis. Conceptually, the status of the sentinel lymph nodes represents the metastatic status of the regional node basin. This allows identification of the subset of patients likely to gain a survival advantage from elective regional lymph node dissection, as recently demonstrated in a report from the Multicenter Selective Lymphadenectomy Trial. This clearly showed a significant survival advantage in sentinel node-positive patients undergoing immediate elective regional lymph node dissection, in comparison to patients who did not have sentinel lymph node biopsy and underwent regional lymphadenectomy only after clinical emergence of nodal disease. ${ }^{2}$ An additional 
benefit of sentinel nodal biopsy lies in sparing those node-negative patients the morbidity (wound separation, infection, hematoma formation and/or lymphedema) associated with unnecessary lymphadenectomy. ${ }^{3,4}$

Since the inception of the sentinel lymph node biopsy procedure, significant strides have been made both in refining the surgical approach to nodal identification and excision and in defining the optimal method of examination of the harvested tissue in the pathology laboratory. ${ }^{5,6}$ Histological evaluation using both routine stains and immunohistochemistry is the practise in most laboratories. Molecular detection of melanocytespecific mRNA by reverse transcription polymerase chain reaction (RT-PCR) is also applied to sentinel lymph node evaluation in many centres. Owing to the exquisitely sensitive nature of this technology, its introduction to clinical practise has revealed a relatively high proportion of sentinel nodes with histologically unexplained RT-PCR positivity.

This has raised the issue of whether detection of tyrosinase mRNA in the absence of histologic evidence of metastatic disease should prompt the surgeon to proceed to elective regional lymphadenectomy. The current practise in this regard is not standardized and is largely institution dependent. In our centre, as in many others, these patients are followed clinically without further intervention. This stems from the knowledge that the RT-PCR technique has a well-documented rate of falsepositivity, attributable in part to the presence capsular nevi in a substantial proportion of sentinel nodes removed from melanoma patients and to other postulated sources of 'melanocytic' markers, including melanophages, certain dendritic and Schwann cells. ${ }^{5,7}$

In this context, we set out to compare the clinical outcome of patients with sentinel lymph node positivity for tyrosinase-mRNA by RT-PCR alone with the outcomes of patients yielding histologic nodal positivity, and of patients whose nodes were negative by both methods. Our objective was to determine whether it is justifiable to withhold elective regional lymph node dissection from the first of these three groups.

\section{Materials and methods}

\section{Patients}

All patients who underwent sentinel lymph node excision for primary cutaneous melanoma at the Queen Elizabeth II Health Sciences Centre between October 1998 and May 2004 were identified retrospectively using the Department of Pathology Laboratory Information System (LIS-Cerner Systems, USA). These mainly included individuals with intermediate thickness melanomas (1.0-4.0 mm) and those with thin $(<1.0 \mathrm{~mm})$ level four melano- mas lacking clinical evidence of regional lymph node involvement. A definitive excision of the primary tumour site was carried out in each case, usually at the time of the sentinel lymph node biopsy procedure. Any case in which the primary tumour was unavailable for pathologic review (ie excised at another institution) was excluded from the study. Patients with histopathological evidence of metastatic melanoma in the sentinel node(s) were offered further treatment with elective regional lymph node dissection as well as consultation with medical oncology, whereas all others were assigned to clinical follow-up alone.

Clinical information was obtained from patients' medical records and from an institutional melanoma patient database developed and maintained by one of the authors (CG). Data collection included the age and gender of the patient, the anatomic site of the primary tumour, length of clinical follow up, the presence and type of disease recurrence and any additional (surgical, medical or radiation) treatment. Recurrence was defined as any clinical, pathological and/or radiological evidence of recurrent local disease, in transit or satellite metastases, regional or distant lymph node involvement and/or systemic or visceral metastases. Duration of follow-up was defined as the interval from the time of the initial pathological tissue diagnosis of melanoma (biopsy or excision) to the most recent clinical evaluation of the patient.

\section{Sentinel Lymph Node Procedure}

Radionucleotide lymphatic mapping was used for preoperative identification of lymph node drainage of the primary tumour. At the time of surgery, injection of the primary tumour site with ${ }^{99 m}$ Te-labeled colloid was followed by lymphoscintigraphy and subsequent intraoperative injection of a non-vital blue dye. Identification and dissection of the sentinel node(s) was aided by both the visible blue color and the radioactive signal detected with a hand held gamma probe.

\section{Sentinel Lymph Node Evaluation}

Processing and pathologic review of the sentinel lymph nodes was based on methods used originally in the Multicentre Selective Lymphadenectomy Trial from the John Wayne Cancer Institute and these are outlined in detail in previous publications by the senior author. ${ }^{8,9}$ Briefly, each harvested node was received fresh in the laboratory and one-quarter was frozen for RNA extraction. Tyrosinase messenger RNA was sought by the RT-PCR technique according to previously published protocols $^{10}$ using appropriate positive and negative controls.

The remaining three-quarters of each node were immediately formalin-fixed and paraffin embedded. 
Six serial sections were produced for histopathological and immunohistochemical analyses. Two sections of each node were stained with hematoxylin and eosin (H\&E). Immunohistochemistry was performed on alternate sections with antibodies for S100 protein (polyclonal rabbit anti-cow antibody, DAKOcytomation, Mississauga, ON, Canada) and HMB45 (anti-human melanosome clone antibody, Ventana, Tucson, AZ, USA) in concert with appropriate positive and negative control sections. Histologic positivity of sentinel lymph nodes was defined as the presence of any evidence of metastatic melanoma by H\&E staining and/or immunohistochemistry. Representative examples of RT-PCR, histologic and immunohistochemical analyses are shown in Figure 1.

The pathology of all primary melanoma and sentinel node specimens was reviewed, at the outset or retrospectively, by one of the authors (NW). The pathologic prognostic features recorded for each primary tumour included depth/thickness (Clark's level and Breslow measurement) and the presence or absence of ulceration.

\section{Statistical Analysis}

Comparative analysis of clinical and pathological factors between groups was performed using a standard $\chi^{2}$ test. Univariate and multivariate logistic regression analysis were performed on primary tumour characteristics, sentinel lymph node status (histologic and molecular) and clinical data to identify prognostic variables.

\section{Results}

\section{Patient and Primary Tumour Characteristics}

The initial computerized search of the LIS identified 169 patients (314 sentinel nodes). Twenty patients were removed from the study owing to insufficient RNA or RT-PCR product for molecular analysis and
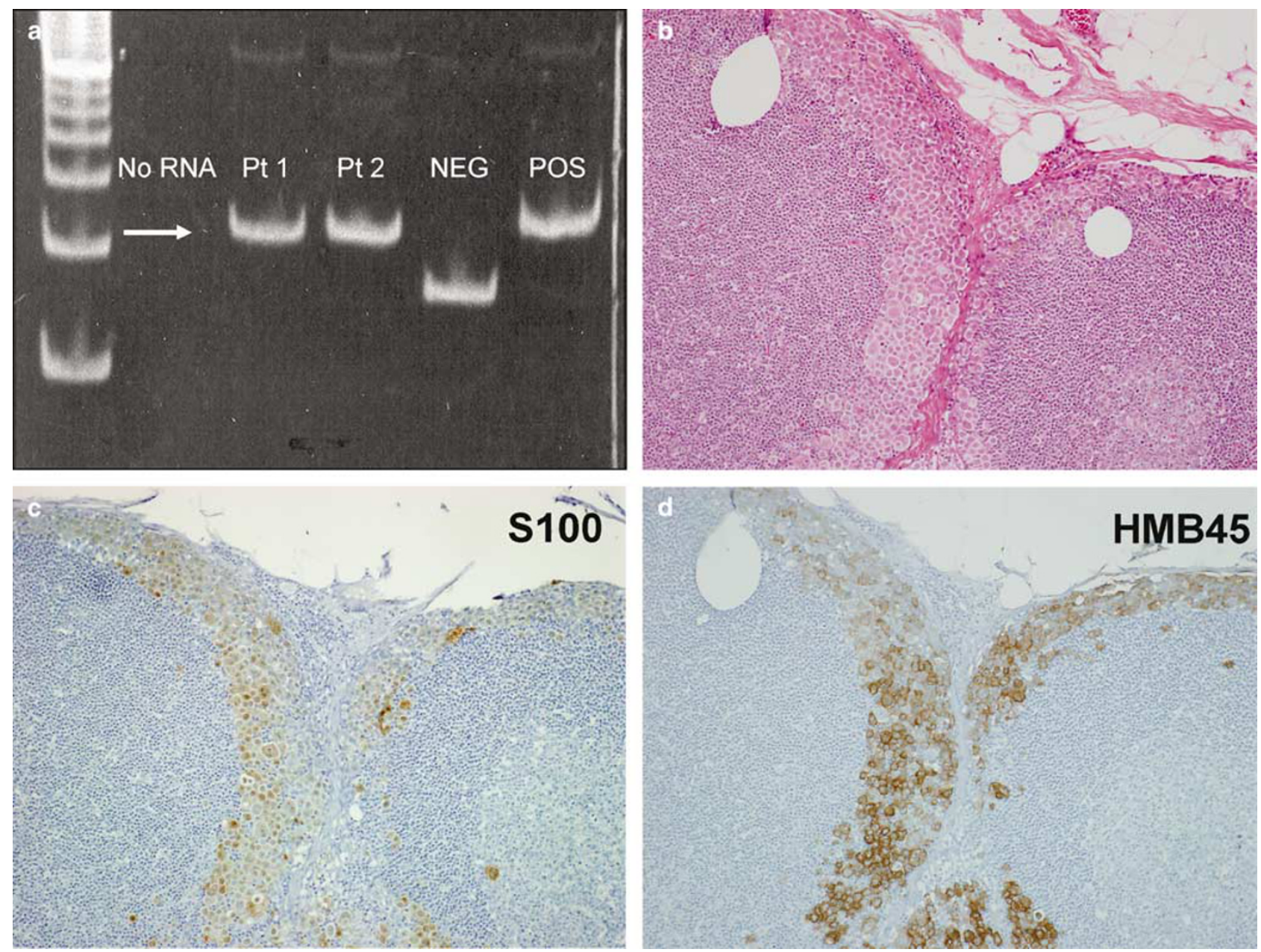

Figure 1 (a) Gel electrophoresis of tyrosinase RT-PCR products: expected 203 base pair (bp) band (white arrow) in patient lanes (Pt 1, Pt 2) and in positive control (POS) lane; lane 2: No RNA (NEG) control; lane 5: Negative control (NEG) with non-specific band; (b) metastatic melanoma in sentinel lymph node (H\&E, medium power); (c) S100 and (d) HMB45 immunohistochemistry of metastatic melanoma seen in (b). 
a further 10 patients were excluded because of incomplete clinical data (ie lost to follow-up). In total, 264 sentinel lymph nodes harvested from 139

Table 1 Clinical characteristics of melanoma patients $(n=139)$

\begin{tabular}{lc}
\hline Characteristic & No. of patients (\%) \\
\hline Gender & $78(56)$ \\
Male & $61(44)$ \\
Female & \\
Age (years) & \\
Mean (range) & \\
All patients & $56.4(14-86)$ \\
Male & $57.4(14-84)$ \\
Female & $55.2(28-86)$ \\
& \\
Site of primary melanoma & \\
Head or neck & $21(15)$ \\
Trunk & $51(37)$ \\
Upper extremity & $39(28)$ \\
Lower extremity & $28(20)$ \\
\hline
\end{tabular}

Table 2 Pathological characteristics of primary tumours $(n=139)$

\begin{tabular}{lr}
\hline Characteristic & Number (\%) \\
\hline Breslow measurement (mm) & \\
$<1.1 \mathrm{~mm}$ & $36(26)$ \\
$1.1-2.0 \mathrm{~mm}$ & $60(43)$ \\
$2.1-3.0 \mathrm{~mm}$ & $25(18)$ \\
$3.1-4.0 \mathrm{~mm}$ & $9(6.5)$ \\
$>4.0 \mathrm{~mm}$ & $9(6.5)$ \\
& \\
Mean & 1.92 \\
Median & 1.40 \\
Range & $0.3-8.0$ \\
& \\
Clark level & \\
II & $3(2.2)$ \\
III & $75(54)$ \\
IV & $58(42)$ \\
V & $3(2.2)$ \\
Ulceration & \\
Yes & $13(9.3)$ \\
No & $125(90)$ \\
Not determined & $1(0.7)$ \\
\hline
\end{tabular}

patients (mean of 1.9; range of 1-8 nodes/patient) were included in the study.

The clinical characteristics of these patients and the pathological characteristics of their primary tumours are shown in Tables 1 and 2, respectively. The mean age of the slightly male predominant study population was 56.4 years (range 14-86). The mean and median Breslow tumour thicknesses were 1.92 and $1.40 \mathrm{~mm}$, respectively, with a corresponding mean and median Clark level of III. There were no significant differences in any of the measured demographic or pathological variables between the original 169 patients and the subset of 139 patients included in this study (data not shown).

\section{Histopathologic and Tyrosinase RT-PCR Analyses}

All sentinel lymph nodes were analyzed by both histopathologic examination (routine H\&E sections and immunohistochemistry) and RT-PCR analysis. Histologic evidence of nodal metastasis was present in $34 / 139$ patients $(24.5 \%)$ and $61 / 139$ (43.9\%) patients demonstrated tyrosinase mRNA positivity by RT-PCR. Sentinel nodes from 13 patients (9.4\%) were found to contain benign capsular nevi.

Patients were then grouped according to nodal status. The first group (70/139 patients, $50.4 \%$ ) had sentinel lymph nodes that were negative by both methods of evaluation (HIS ${ }^{\mathrm{NEG}} / \mathrm{PCR}^{\mathrm{NEG}}$ ). The second group included 35 cases $(25.2 \%)$ with RT-PCR positivity for tyrosinase mRNA, where metastatic disease was undetected by histopathologic means (HIS $^{\mathrm{NEG}} / \mathrm{PCR}^{\mathrm{POS}}$ ). Benign capsular nevi were found in five of these 35 cases ( $\mathrm{HIS}^{\mathrm{NEG}} / \mathrm{PCR}^{\mathrm{POS}} / \mathrm{CN}^{\mathrm{POS}}$ ) leaving 30 of the 139 patients $(21.6 \%)$ with unexplained RTPCR positivity $\left(\mathrm{HIS}^{\mathrm{NEG}} / \mathrm{PCR}^{\mathrm{POS}} / \mathrm{CN}^{\mathrm{NEG}}\right.$ ). The final group of 34 patients demonstrated histopathologic evidence of metastatic disease, of which 26 showed concurrent RT-PCR positivity for tyrosinase mRNA (HIS ${ }^{\mathrm{POS}} / \mathrm{PCR}^{\mathrm{POS}}$ ). Eight patients had histopathological evidence of metastatic melanoma that was not detected by RT-PCR (HIS ${ }^{\mathrm{POS}} / \mathrm{PCR}^{\mathrm{NEG}}$ ).

Using histologic positivity as the 'gold standard', the calculated sensitivity and specificity of RT-PCR detected tyrosinase mRNA was 76 and $67 \%$, respec-

Table 3 Patient groups defined by SLN status

\begin{tabular}{|c|c|c|c|c|c|c|c|}
\hline \multirow[t]{2}{*}{ Patient group } & \multirow{2}{*}{$\begin{array}{c}\text { Patient number } \\
(\%)\end{array}$} & \multicolumn{2}{|c|}{ Breslow thickness (mm) } & \multirow[t]{2}{*}{ Ulceration (\%) } & \multicolumn{2}{|c|}{ Months of follow-up } & \multirow[t]{2}{*}{ Recurrence (\%) } \\
\hline & & Mean & Median (range) & & Mean & Median (range) & \\
\hline $\mathrm{HIS}^{\mathrm{POS}} / \mathrm{PCR}^{\mathrm{POS}}$ & $26(19)$ & 3.13 & $2.35(0.7-7.9)$ & $6(23)$ & 22.5 & $22.0(3-48)$ & $16(62)$ \\
\hline $\mathrm{HIS}^{\mathrm{POS}} / \mathrm{PCR}^{\mathrm{NEG}}$ & $8(5.8)$ & 2.52 & $2.25(1.3-4.3)$ & $0(0)$ & 29.9 & $25.5(4-61)$ & $6(75)$ \\
\hline $\mathrm{HIS}^{\mathrm{NEG}} / \mathrm{PCR}^{\mathrm{NEG}}$ & $70(50)$ & 1.46 & $1.20(0.3-4.0)$ & $3(4.3)$ & 30.6 & $32.0(1-74)$ & $7(10)$ \\
\hline $\mathrm{HIS}^{\mathrm{NEG}} / \mathrm{PCR}^{\mathrm{POS}} / \mathrm{CN}^{\mathrm{NEG}}$ & $30(22)$ & 1.87 & $1.40(0.7-8.0)$ & $4(13)$ & 24.8 & $19.5(4.5-74)$ & $2(7.2)$ \\
\hline $\mathrm{HIS}^{\mathrm{NEG}} / \mathrm{PCR}^{\mathrm{POS}} / \mathrm{CN}^{\mathrm{POS}}$ & $5(3.6)$ & 1.30 & $1.30(0.9-1.9)$ & $0(0)$ & 28.0 & $26.0(9-56)$ & $0(0)$ \\
\hline
\end{tabular}

Abbreviations: $\mathrm{CN}^{\mathrm{POS}}$, capsular nevus detected; $\mathrm{CN}^{\mathrm{NEG}}$, capsular nevus not detected; $\mathrm{HIS}^{\mathrm{NEG}}$, negative by histological/immunohistochemical evaluation; HIS ${ }^{\text {POS }}$, positive by histological/immunohistochemical evaluation; PCR ${ }^{\text {POS }}$, tyrosinase mRNA detected by RT-PCR; PCR ${ }^{\text {NEG }}$, tyrosinase mRNA not detected by RT-PCR; SLN, sentinel lymph node. 
tively, with a positive predictive value (PPV) of $43 \%$ and negative predictive value (NPV) of $90 \%$.

\section{SLN Status and Clinical Outcome}

The mean duration of follow-up for all patients was 27.7 months, with a median of 25.0 (range 1-74) months. During this time, recurrence of all forms (local, nodal and/or systemic) had developed in 31 of 139 patients $(22.3 \%)$. Table 3 compares the overall recurrence rates, duration of follow-up and primary tumour characteristics between each group of patients as defined by histopathologic and RT-PCR status of sentinel lymph nodes.

Recurrence rates were similar among patients with positive histopathology, irrespective of RT-PCR status (HIS ${ }^{\mathrm{POS}} / \mathrm{PCR}^{\mathrm{POS}} 62.5 \%$ and $\mathrm{HIS}^{\mathrm{POS}}$ / PCR $^{\text {NEG }} 75 \%$ ). Recurrence developed in seven of 70 patients $(10 \%)$ in the HIS ${ }^{\mathrm{NEG}} / \mathrm{PCR}^{\mathrm{NEG}}$ group and in two of $30(7.2 \%)$ of the HIS ${ }^{\mathrm{NEG}} / \mathrm{PCR}^{\mathrm{POS}} / \mathrm{CN}^{\mathrm{NEG}}$ group. Statistically, recurrence rates were similar between the two HIS ${ }^{\text {NEG }}$ and between the two HIS ${ }^{\text {POS }}$ groups (each $P>0.05$ ). Furthermore, there were significant differences in disease recurrence rates $(P<0.0001)$ when comparing HIS ${ }^{\text {NEG }}$ groups to HIS ${ }^{\text {POS }}$ groups. No recurrence was seen in the $\mathrm{HIS}^{\mathrm{NEG}} / \mathrm{PCR}^{\mathrm{POS}} / \mathrm{CN}^{\mathrm{POS}}$ group $(n=5)$. It was decided to remove these patients from subsequent statistical analysis on the

Table 4 Prognostic indicators of recurrence: univariate analysis of clinical and pathological characteristics of melanoma patients $(n=134)$

\begin{tabular}{lc}
\hline Characteristic & P-value \\
\hline Clinical & \\
Gender & \\
Age & 0.0912 \\
& 0.3679 \\
Primary tumour & 0.6424 \\
Location & $<0.0001$ \\
Breslow thickness & 0.0178 \\
Clark level & 0.0636 \\
Ulceration & \\
SLN status (histologic positivity) & $<0.0001$
\end{tabular}

Abbreviation: SLN, sentinel node status. basis that the capsular nevi were responsible for tyrosinase mRNA expression.

Univariate logistical regression analysis was performed to determine which demographic, primary tumour and nodal factors were prognostic indicators of overall recurrence (Table 4). In addition to the primary tumour thickness (Breslow measurement and Clark level), the histologic status of sentinel lymph nodes was the only other significant prognostic indicator of disease recurrence in the population studied. When these three variables were examined in a multiple logistic regression analysis, only the Breslow thickness $(P=0.0193)$ and nodal positivity $(P<0.0001)$ remained as independent significant prognostic factors. The Clark level was no longer significant $(P=0.169)$. Patient gender, age, tumour location and/or presence of ulceration did not significantly impact on recurrence. The number of primary tumours displaying ulceration was small (13 in total). The presence of ulceration of the primary melanoma did not correlate with positive histologic sentinel lymph node status $(P=0.0849$, standard Fisher's exact test).

The type of disease recurrence (local, regional, distant, dead of disease) in relation to sentinel lymph node status is presented in Table 5. Although the sample sizes were relatively small in this analysis, there was continuation of the general trend of higher recurrence rates in HIS ${ }^{\mathrm{POS}}$ patients that were not affected by RT-PCR status. Death from malignant melanoma occurred in $11 / 34(32.3 \%)$ of HIS $^{\text {POS }}$ patients and in $5 / 100(5 \%)$ of HIS ${ }^{\text {NEG }}$ patients. Melanoma-related deaths were seen in both the $\mathrm{HIS}^{\mathrm{NEG}} / \mathrm{PCR}^{\mathrm{POS}} / \mathrm{CN}^{\mathrm{NEG}}$ and $\mathrm{HIS}^{\mathrm{NEG}} / \mathrm{PCR}^{\mathrm{NEG}}$ groups with similar frequency (4.3 vs 6.7\%). Multivariate analysis revealed statistically significant differences between the HIS ${ }^{\mathrm{NEG}}$ and HIS ${ }^{\mathrm{POS}}$ groups within all categories of recurrence $(P=0.0019$ $P=0.0184$ ). Breslow thickness was a significant predictor only for overall recurrence and regional lymph node involvement $(P=0.0026)$.

\section{Discussion}

After grouping our patients according to sentinel lymph node status and then examining the clinical

Table 5 Types of disease recurrence among patient groups defined by SLN status $(n=134)$

\begin{tabular}{|c|c|c|c|c|c|}
\hline Group & All recurrence (\%) & $\operatorname{Local}^{\mathrm{a}}(\%)$ & Regional $(\%)$ & Distant $^{\mathrm{c}}(\%)$ & $D O D(\%)$ \\
\hline $\mathrm{HIS}^{\mathrm{POS}} / \mathrm{PCR}^{\mathrm{POS}}(n=26)$ & $16(62)$ & $6(23)$ & $9(35)$ & $12(46)$ & $10(38)$ \\
\hline $\mathrm{HIS}^{\mathrm{POS}} / \mathrm{PCR}^{\mathrm{NEG}}(n=8)$ & $6(75)$ & $4(50)$ & $3(38)$ & $2(25)$ & $1(12)$ \\
\hline $\mathrm{HIS}^{\mathrm{NEG}} / \mathrm{PCR}^{\mathrm{NEG}}(n=70)$ & 7 (10) & $3(4.3)$ & $3(4.3)$ & $4(5.7)$ & $3(4.3)$ \\
\hline $\mathrm{HIS}^{\mathrm{NEG}} / \mathrm{PCR}^{\mathrm{POS}} / \mathrm{CN}^{\mathrm{NEG}}(n=30)$ & $2(7.2)$ & $0(0)$ & $1(3.3)$ & $2(6.7)$ & $2(6.7)$ \\
\hline
\end{tabular}

Abbreviations: SLN, sentinel node status; DOD, dead of disease.

${ }^{\mathrm{a}}$ Local recurrence, satellite metastases and/or in transit metastases.

${ }^{\mathrm{b}}$ Regional lymph node metastases.

${ }^{\mathrm{c}}$ Distant lymph node, systemic and/or visceral metastases. 
Table 6 Summary of patient groups based on SLN status in recently published study populations

\begin{tabular}{|c|c|c|c|c|c|c|c|c|c|c|c|}
\hline \multirow[t]{2}{*}{ Reference } & \multirow{2}{*}{$\begin{array}{l}\text { Population } \\
\text { (n) }\end{array}$} & \multirow{2}{*}{$\begin{array}{c}\text { Median (mean) } \\
\text { duration of } \\
\text { follow up } \\
\text { (months) }\end{array}$} & \multicolumn{3}{|c|}{ SLN status of study population } & \multicolumn{3}{|c|}{ Recurrence rates } & \multirow[t]{2}{*}{$C N$} & \multirow{2}{*}{$\begin{array}{l}\text { RT-PCR } \\
\text { Markers }\end{array}$} & \multirow{2}{*}{$\begin{array}{c}\text { Median (mean) } \\
\text { Breslow } \\
\text { thickness } \\
\text { (mm) }\end{array}$} \\
\hline & & & $\begin{array}{l}H I S^{N E G} / \\
P C R^{N E G}\end{array}$ & $\begin{array}{l}H I S^{N E G} / \\
P C R^{P O S}\end{array}$ & $\begin{array}{c}H I S^{P O S} / P C R^{P O S} \\
o r \\
H I S^{P O S} / P C R^{N E G}\end{array}$ & $\begin{array}{l}H I S^{N E G} / \\
P C R^{N E G}\end{array}$ & $\begin{array}{l}H I S^{N E G} / \\
P C R^{P O S}\end{array}$ & $\begin{array}{c}H I S^{P O S} / P C R^{P O S} \\
o r \\
H I S^{P O S} / P C R^{N E G}\end{array}$ & & & \\
\hline Li et al $(2000)^{11}$ & 233 & $(24)$ & $29 \%$ & $49 \%$ & $22 \%$ & $1.6 \%$ & $10 \%$ & $37 \%$ & DNP & Tyrosinase & $(2.18)$ \\
\hline Rimoldi et al $(2003)^{12}$ & 57 & 36 & $29 \%$ & $33 \%$ & $28 \%$ & $9 \%$ & $16 \%$ & $38 \%$ & $11 \%$ & Tyrosinase & $1.9(2.46)$ \\
\hline Ribuffo et al (2003) ${ }^{13}$ & 134 & $(42)$ & $37 \%$ & $52 \%$ & $11 \%$ & DNP & DNP & DNP & $3.4 \%$ & $\begin{array}{l}\text { Tyrosinase } \\
\text { MART1 }\end{array}$ & 1.82 \\
\hline Ulrich et al $(2004)^{14}$ & 322 & 37 & $77 \%$ & $12 \%$ & $11 \%$ & $8 \%$ & $26 \%$ & $44 \%$ & $0 \%$ & Tyrosinase & $1.22(2.11)$ \\
\hline Kammula et al $(2005)^{15 a}$ & 112 & $\begin{array}{l}42 \\
67\end{array}$ & $35 \%$ & $52 \%$ & $13 \%$ & $\begin{array}{c}0 \% \\
15 \%\end{array}$ & $\begin{array}{l}14 \% \\
24 \%\end{array}$ & $\begin{array}{l}53 \% \\
67 \%\end{array}$ & $11 \%$ & Tyrosinase & $2.2(2.6)$ \\
\hline Romanini et al $(2005)^{16}$ & 124 & 30 & $69 \%$ & $13 \%$ & $19 \%$ & $9.4 \%$ & $31 \%$ & $60 \%$ & DNP & $\begin{array}{l}\text { Tyrosinase } \\
\text { MART1 }\end{array}$ & DNP \\
\hline Mangas et al $(2006)^{17}$ & 180 & 45 & $31 \%$ & $48 \%$ & $21 \%$ & $5 \%$ & $10 \%$ & $30 \%$ & $2.8 \%$ & Tyrosinase & $1.36(2.19)$ \\
\hline Scoggins et al $(2006)^{18 \mathrm{~b}}$ & 1446 & 30 & $75 \%$ & $25 \%$ & - & $10 \%$ & $11 \%$ & - & DNP & $\begin{array}{l}\text { Tyrosinase } \\
\text { MART1 } \\
\text { MAGE3 } \\
\text { GP100 }\end{array}$ & $1.6(2.1)$ \\
\hline Present study & 134 & $25(27)$ & $50 \%$ & $22 \%$ & $25 \%$ & $10 \%$ & $7.2 \%$ & $65 \%$ & $9.4 \%$ & Tyrosinase & $1.40(1.92)$ \\
\hline
\end{tabular}

Abbreviations: CN, capsular nevi; DNP, data not published; SLN, sentinel lymph node status.

${ }^{\mathrm{a}}$ Recurrence rates in this study were calculated at two different time points (42 and 67 months).

${ }^{\mathrm{b}} \mathrm{PCR}^{\mathrm{POS}}$ in this study was defined as the detection of tyrosinase mRNA in addition to at least one other marker by RT-PCR. 
outcome in each group, a clear difference emerged in the overall recurrence rates between the HIS ${ }^{\mathrm{POS}}$ and HIS ${ }^{\mathrm{NEG}}$ groups, reiterating the well-established independent prognostic significance of histopathologic evidence of nodal metastasis. We found the same to be true of the Breslow thickness of the primary melanoma, in accordance with existing data in the literature. In contrast to previous reports, ulceration of the primary tumour did not carry independent prognostic significance in our population. The relatively small number of ulcerated tumours in our study $(9.3 \%)$ may have resulted in a lack of statistical power to demonstrate significance.

The clinical outcome of patients in our study with positive results on molecular studies alone (HIS ${ }^{\mathrm{NEG} /}$ $\mathrm{PCR}^{\mathrm{POS}}$ ) was comparable to that of patients whose sentinel nodes were negative by both assessments $\left(\right.$ HIS $^{\mathrm{NEG}} / \mathrm{PCR}^{\mathrm{NEG}}$ ) and was significantly better than that observed in HIS $^{\mathrm{POS}}$ patients. In addition, the relative rates of specific types of recurrence (local, regional, systemic and melanoma related-mortality) between the $\mathrm{HIS}^{\mathrm{NEG}} / \mathrm{PCR}^{\mathrm{POS}}$ and $\mathrm{HIS}^{\mathrm{NEG}} / \mathrm{PCR}^{\mathrm{NEG}}$ groups were similar, these being significantly lower than those observed in the HIS ${ }^{\mathrm{POS}}$ group. These results support the current conservative approach to management of $\mathrm{HIS}^{\mathrm{NEG}} / \mathrm{PCR}^{\mathrm{POS}}$ patients, sparing these patients a significant number of unnecessary lymphadenectomy procedures with its associated morbidity.

Our results differ from earlier published reports on this topic ${ }^{11-16}$ showing that molecular detection of metastasis in sentinel lymph nodes was of prognostic significance. These studies demonstrated recurrence rates in $\mathrm{HIS}^{\mathrm{NEG}} / \mathrm{PCR}^{\mathrm{POS}}$ patients that were intermediate between $\mathrm{HIS}^{\mathrm{NEG}} / \mathrm{PCR}^{\mathrm{NEG}}$ and $\mathrm{HIS}^{\mathrm{POS}}$ populations, ranging from 10 to $31 \%$. In contrast, the two most recent studies, ${ }^{17,18}$ including the largest randomized prospective trial to date (The Sunbelt Melanoma Trial) support our findings. The recurrence rate in $\mathrm{HIS}^{\mathrm{NEG}} / \mathrm{PCR}^{\mathrm{POS}}$ patients in these reports and in our study collectively was $7.2-11 \%$, substantially lower than those previously reported, and not significantly greater than in HIS ${ }^{\mathrm{NEG}} / \mathrm{PCR}^{\mathrm{NEG}}$ patients. Table 6 compares the populations and results of these reports ${ }^{11-18}$ with those of the present study. To our knowledge, ours is the first analysis with a retrospective design and relatively short duration of follow-up to show a lack of prognostic significance for tyrosinase expression in sentinel nodes.

It could be argued that the significance of our findings is limited by the short duration of follow-up (median 25 months). However, this time period is within the spectrum of those reported by others. A recent prospective study ${ }^{15}$ showed that a statistically significant difference in rate of recurrence in the $\mathrm{HIS}^{\mathrm{NEG}} / \mathrm{PCR}^{\mathrm{POS}}$ group compared to $\mathrm{HIS}^{\mathrm{NEG}} / \mathrm{PCR}^{\mathrm{NEG}}$ patients 14 vs $0 \%, P<0.05$ found at 42 months of follow-up was lost by 67 months (24 vs $15 \%, P=0.25$ ). Given that the median time to relapse in the $\mathrm{HIS}^{\mathrm{NEG}} / \mathrm{PCR}^{\mathrm{NEG}}$ group was approximately 10 months later than in HIS ${ }^{\mathrm{NEG} /}$ $\mathrm{PCR}^{\mathrm{POS}}$ patients, the authors concluded that the $\mathrm{PCR}^{\mathrm{POS}}$ patients developed earlier onset recurrence. If this is true, then it is likely that our findings would not change with a longer duration of follow-up. There is little doubt that additional work is required in this field and it can be expected that larger multicenter studies with a greater spectrum of clinical follow-up will yield the answer.

Of patients in our study without histopathological or molecular evidence of metastatic disease in sentinel lymph nodes, $10 \%$ had recurrent disease within the follow-up period. This is comparable to recurrence rates in $\mathrm{HIS}^{\mathrm{NEG}} / \mathrm{PCR}^{\mathrm{NEG}}$ patients reported in prior studies, which ranged from 1.6 to $15 \% .{ }^{11-15}$ This apparent paradoxical pattern of disease progression may in part be explained by metastasis of some melanomas via the blood stream as opposed to the lymphatic system. Of the seven $\mathrm{HIS}^{\mathrm{NEG}} / \mathrm{PCR}^{\mathrm{NEG}}$ patients in this study with recurrence, only one patient developed distant metastases and died of metastatic melanoma without local or regional LN involvement. This one case may reflect haematogenous rather than lymphatic spread. The remaining six patients likely represent limitations in tissue sampling, evaluation methodologies and/or human interpretative error.

The prognostic significance of histopathological evidence of metastases in the sentinel lymph nodes of melanoma patients is beyond doubt and its inclusion as a valid staging parameter is well accepted. Additional work is required to address the significance of positive findings using highly sensitive molecular methods, such as RTPCR, in this context. For example, it is open to question whether some of the 'melanoma-specific' molecular markers in current use are truly melanoma-specific or whether they can be found in other cells such as Schwann cells or melanophages. Issuing from this is the question of whether positivity for multiple, rather than single, molecular markers carries more significance in predicting disease recurrence. Furthermore, whether a critical volume of metastatic melanoma cells in sentinel nodes has a relationship to disease recurrence is unknown and this may have a bearing on whether a defined threshold of positivity for melanoma markers, detectable by RT-PCR, would be of value. The substantial number of patients with $\mathrm{HIS}^{\mathrm{NEG}} / \mathrm{PCR}^{\mathrm{POS}}$ sentinel nodes makes it imperative to resolve these issues, which are currently a focus of study by others. Our results indicate that, in the short-term, disease recurrence in patients with single marker positivity for tyrosinase mRNA is no different from that in patients whose nodes are negative by both methodologies. Given the conflicting data in the literature all available data on the subject require scrutiny. 


\section{Acknowledgements}

We thank Wade Blanchard and Melanie Abeysundera of the Department of Mathematics and Statistics, Dalhousie University, Halifax for their contributions to the statistical analysis in this study.

\section{References}

1 Balch CM, Buzaid AC, Soong S-J, et al. Final version of the american joint committee on cancer staging system for cutaneous melanoma. J Clin Oncol 2001;19: 3635-3648.

2 Morton DL, Thompson JF, Cochran AJ, et al. Sentinelnode biopsy or nodal observation in melanoma. N Engl J Med 2006;355:1307-1317.

3 Chakera AH, Drzewiecki KT, Eigtved A, et al. Sentinel node biopsy for melanoma: a study of 241 patients. Melanoma Res 2004;14:521-526.

4 Morton DL, Cochran AJ, Thompson JF, et al. Sentinel Node Biospy for Early-Stage Melanoma-Accuracy and morbidity in MSLT-1, an International Multicenter Trial. Ann Surg 2005;242:302-313.

5 Abrahamsen HN, Hamilton-Dutoit SJ, Larsen J, et al. Sentinel lymph nodes in malignant melanomaextended histopathologic evaluation improves diagnostic precision. Cancer 2004;100:1683-1691.

6 Spanknebel K, Coit DG, Bieligk SC, et al. Characterization of micrometastatic disease in melanoma sentinel lymph nodes by enhanced pathology-recommendations for standardizing pathologic analysis. Am J Surg Pathol 2005;29:305-317.

7 Starz H. Pathology of the sentinel lymph node in melanoma. Semin Oncol 2004;3:357-362.

8 Fontaine D, Parkhill WP, Greer W, et al. Nevus Cells in lymph nodes-an association with congenital cutaneous nevi. Am J Dermatopathol 2002;24:1-5.

9 Fontaine D, Parkhill WP, Greer W, et al. Partial Regression of Primary Cutaneous Melanoma-is there an association with sub-clinical sentinel lymph node metastasis. Am J Dermatopathol 2003;25:371-376.
10 Van der Velde-Zimmerman D, Roijers JF, BouwensRombouts A, et al. Molecular test for the detection of tumor cells in blood and sentinel lymph nodes of melanoma patients. Am J Pathol 1996;149: 759-764.

11 Li W, Stall A, Shivers SC, et al. Clinical relevance of molecular staging for melanoma-comparison of RTPCR and Immunohistochemistry staining on sentinel lymph nodes of patients with melanoma. Ann Surg 2000;231:795-803.

12 Rimoldi D, Lemoine R, Kurt A-M, et al. Detection of micrometastases in sentinel lymph nodes from melanoma patients: direct comparison of multimarker molecular and immunopathological methods. Melanoma Res 2003;13:511-520.

13 Ribuffo D, Gradilone A, Vonella $\mathrm{M}$, et al. Prognostic significance of reverse transcriptase-polymerase chain reaction-negative sentinel lymph nodes in malignant melanoma. Ann Surg Oncol 2003;10: 396-402.

14 Ulrich J, Bonnekoh B, Bockelmann R, et al. Prognostic significance of detecting micrometastases by tyrosinase RT/PCR in sentinel lymph node biopsies: lessons from 322 consecutive melanoma patients. Eur J Cancer 2004;40:2812-2819.

15 Kammula US, Ghossein R, Bhattacharya S, et al. Serial follow-up of reverse transcriptase-polymerase reaction-staged sentinel lymph nodes from melanoma patients. J Clin Oncol 2005;22: 3989-3996.

16 Romanini A, Manca G, Pellegrino D, et al. Molecular staging of the sentinel lymph node in melanoma patients: correlation with clinical outcome. Ann Oncol 2005;16:1832-1841.

17 Mangas C, Hilari JM, Paradelo C, et al. Prognostic significance of molecular staging study of sentinel lymph nodes by reverse transcriptase-polymerase chain reaction for tyrosinase in melanoma patients. Ann Surg Oncol 2006;13:910-918.

18 Scoggins CH, Ross MI, Reintgen DS, et al. Prospective multi-institutional study of reverse transcriptase polymerase chain reaction for molecular staging of melanoma. J Clin Oncol 2006;24:1849-1857. 